\title{
Loop-Mediated Isothermal Amplification (LAMP) Assay for GMO on: Recent Progresses and Future Perspectives
}

\author{
Rong Li1,2, Cong Wang², Lilian Ji1,3*, Xiangxiang Zhao3, Man Liu4, Dabing Zhang2,5, \\ Jianxin Shi ${ }^{2,4^{*}}$ \\ ${ }^{1}$ School of Tourism and Culinary Science, Yangzhou University, Yangzhou, China \\ ${ }^{2}$ Shanghai Jiao Tong University-University of Adelaide Joint Centre for Agriculture and Health, School of Life \\ Sciences and Biotechnology, Shanghai Jiao Tong University, Shanghai, China \\ ${ }^{3}$ School of Life Science, Huaiyin Normal University, Huai'an, China \\ ${ }^{4}$ Shanghai Ruifeng Agro-Biotechnology Co., Ltd., Shanghai, China \\ ${ }^{5}$ Plant Genomics Center, School of Agriculture, Food and Wine, University of Adelaide, Adelaide, Australia \\ Email: ${ }^{2}$ ill2663@sina.com, ${ }^{*}$ jianxin.shi@sjtu.edu.cn
}

Received 6 January 2015; accepted 21 January 2015; published 26 January 2015

Copyright (C) 2015 by authors and OALib.

This work is licensed under the Creative Commons Attribution International License (CC BY).

http://creativecommons.org/licenses/by/4.0/

(c) (i) Open Access

\begin{abstract}
As more and more genetically modified (GM) crops are approved for commercialization and planting, safety issues of GM crops have become hot topics worldwide. For both regulatory and academic purposes, development of rapid, economic and effective on-site detection methods for GM components is indispensible. Up to now, the most effective and sensitive techniques used for GMO detection are based on polymerase chain reaction (PCR). PCR method needs expensive, heavy instruments and gel electrophoresis. Therefore, it is commonly used in laboratory test, and unsuitable for on-site detection. Loop-mediated isothermal amplification (LAMP), an isothermal nucleic acid amplification technique, has been extensively used in many areas such as food safety and clinic diagnosis. Advantageous characteristics of LAMP, such as high specificity and sensitivity, simple operation, low cost, eye visualization, particularly free of special equipment, render it with high potential to be used for GMO on-site detection. In this review, we summarized current status of the application of LAMP in GMO detection, and discussed possible improvements needed for its adaptability regarding to on-site GMO detection. Hopefully, the information present here would facilitate the practical risk assessment of GMO.
\end{abstract}

\section{Keywords}

Biotechnology, Genetically Modified Organism (GMO), Loop-Mediated Isothermal Amplification (LAMP), Polymerase Chain Reaction (PCR), Safety

\footnotetext{
${ }^{*}$ Corresponding authors.
}

How to cite this paper: Li, R., Wang, C., Ji, L.L., Zhao, X.X., Liu, M., Zhang, D.B. and Shi, J.X. (2015) Loop-Mediated Isothermal Amplification (LAMP) Assay for GMO Detection: Recent Progresses and Future Perspectives. Open Access Library Journal, 2: e1264. http://dx.doi.org/10.4236/oalib.1101264 


\section{Introduction}

Commercialization of genetically modified (GM) crops started in 1996 in USA, and now GM crops are increasingly gained acceptance worldwide, which makes GM crops the fastest adopted crop technology in the history of modern agriculture and biotechnology [1]. Along with the commercialization process of GM crops, consumers' concerns about their biosafety are concurrently increasing. In addition, the anti-GM groups, such as Greenpeace and Gene Campaign, are strengthening the possible growth of unauthorized varieties and cross-contamination of GM crops [2]. To resolve these issues, legislations concerning GM crop commercialization have been adopted. Although legislations differ from country to country, some issues are common. With increased acceptance of GM organisms (GMO) amongst consumers and society, traceability and labeling are desperately necessary. To comply with these legislations, detection methods are needed [3]. So far, various detection methods have been developed for GMO detection, which have been reviewed elsewhere previously [3]-[6]. Among them, loop-mediated isothermal amplification (LAMP) emerges as one of novel potential alternatives for quick and economic GMO detection [7].

\subsection{Global Status of GM Crops}

According to a recent report released from International Service for The Acquisition of Agri-Biotech Applications (ISAAA), ISAAA Brief 46-2013 [8], GM crop planting area increased every single year between 1996 to 2013, and the total planting area increased by more than 100-fold from 1.7 million hectares in 1996 to over 175 million hectares in 2013. Eight developed countries and 19 developing countries planted GM crops in 2013, and developing countries planted more hectares than developed ones for the second consecutive years. The numbers of farmers that plant GM crops rose up to a record of 11 million. The top 5 countries planting GM crops included USA, Brazil, Argentina, India and Canada; each planted more than 10 million hectares of GM crops. GM crops contributed to food security, sustainability, and the environment substantially by increasing yield, providing better environment, saving pesticides usage, reducing $\mathrm{CO}_{2}$ emission, conserving biodiversity, and alleviating poverty [8]. Since 1994, in total 62 countries in the world (including 27 European Union member countries) have approved the planting and/or importing GM crops for food and feed, covering 2833 approvals for 336 GM events in 27 plant species. Corn topped the approved events (130 events in 27 countries), which is followed by cotton (49 events in 22 countries), potato (31 events in 10 countries), canola (30 events in 12 countries) and soybean (27 events in 26 countries). Although planted GM crops mostly harbor traits of herbicide tolerance or insect resistance, notably, 13 countries planted 47 million hectares of stacked GM crops in 2013, accounting for $27 \%$ of global planting area [8], which indicating a more complexity of GM crop production.

\subsection{GMO Safety Issues and Regulation}

Along with the rapid adoption of GM crops in modern agriculture, the debate over the biosafety of GMOs has never ceased. Public fears about possible food and environment risk of GM crops, which is a matter of concern. It is well-known that there is no biotechnology that is absolutely safe; therefore, GM crop could bring their risks together with their benefits to the environment and human being. In an attempt to relieve public concerns about GM food safety, a series of regulations and legislations have been established in more than 50 countries and regions to strengthen the management of GMOs and label the products containing GM contents [9]. In many countries, the labeling of grains, feed and food derivatives is compulsory if the GMO content exceeds a certain threshold level, which differs depending on countries and regions, and on product types in those countries [6]. To implement the monitoring and labeling of GM crops, detection strategies and techniques are needed. Because the number and complexity of GM crops are remarkably increasing, development of pragmatic, economic, timing-saving and, qualitative and quantitative detection methods is certainly needed.

\subsection{Detection Methods Currently Used}

Generally, the detection methods for GM crops and their products were developed to detect the presence of ei- 
ther protein or nucleic acid sequence of the exogenous insertion. Protein-based detection methods include enzyme-linked immunosorbent assay (ELISA) and lateral flow strips (LFS) [10], while DNA-based methods consist of conventional polymerase chain reaction (PCR), quantitative real-time PCR, LAMP, DNA chip, digital PCR, and so on [5] [10] [11].

The intrinsic drawbacks of protein-based detection methods have been well documented in many excellent reviews [3] [5] [10] [12], which, together with low sensitivity and cross interaction, limited their use in GMO detection. LFS can be used as an economic and quick on-site GMO detection method, but it is not suitable for on-site detection of GM components in processed products. In addition, LFS detects only the presence of the expressed exogenous protein, therefore, it can not distinct samples with same exogenous proteins and serves only for qualitative screening. Even though, low sensitivity due to sample matrix effect, low specificity due to the lack of suitable antibody and false positive due to the non-specific binding often affect the detection result [5]. As compared with protein-based methods, DNA-based methods, such as PCR or real-time quantitative PCR (RT-qPCR), are the most widely used analytical approaches in GMO detection due to their high accuracy, reliability, sensitivity, and re-productivity [3] [5] [10] [11]. Although PCR based methods dominated the current GMO detection, it is constrained mainly in detection laboratory because of its high-precision, heavy, and expensive apparatus and complex affiliated procedures, which restrict their use for on-site detection [13]. Therefore, practical, feasible, low cost, and highly efficient on-site detection methods based on DNA, alternatives to PCR, need to be developed.

\subsection{LAMP: A Novel GMO Detection Method}

Among these analytical methods for GMO detection, LAMP, one PCR-free method for isothermal nucleic acid amplification, developed by Notomi et al. in 2000 [14], has been paid more attention. Due to the nature of fast, accurate, and cost-effective, LAMP has been widely and mainly applied in the rapid molecular diagnosis of pathogens, bacteria and viruses, GMO ingredients, tumor detection, and embryo sex identification [15]-[17]. The number of studies about LAMP has been increasing every year. Its principle and application have been well described by many reviews [15]-[17], which therefore will not be included in this paper.

As an alternative to normal PCR, the amplification of target DNAs with LAMP is obviously faster than conventional PCR-based technologies. It is less sensitive to inhibitors [18] [19], no need of complicate equipment, simple to operate, visual result, and easy to be developed into commercially available detection kits [20] [21]. All those advantageous characteristics make LAMP an ideal choice of on-site GMO detection method. However, why is it still in laboratory experiment stage? This review will focus first on its current applications, disadvantaged features, and then future improvements needed for LAMP to be adopted for on-site GMO detection.

\section{The Application of LAMP in GMO Detection}

Because of its advantages, LAMP has been tested for its applicability in GMO detection. Generally, it is used mainly in following three sections (for detailed information, please refer to Table 1).

\subsection{The Screening of GMO}

In this case, the LAMP targets mainly the general exogenous elements; therefore, the developed method can be used to screen for the presence of GMO components. A GMO screening method using LAMP targeting GMelement Cauliflower Mosaic Virus 35S (P-35S) was first reported by Fukuta et al. (2004) [22]. So far, LAMP methods for T-nos, P-35S, P-nos [23] and the pat marker gene [24] have been developed for GMO element screening in the laboratory.

Some LAMP assay also targets exogenous genes. For example, LAMP for cry1Ab gene in GM rice has been recently developed and proved to be better than qPCR detection [25]. In addition, cry2Ab and cry3A genes, two of the most important insect-resistant exogenous genes, have been detected by visual LAMP within 60 min at an isothermal condition of $63^{\circ} \mathrm{C}$. And the sensitivity of LAMP assay was five copies of haploid genomic DNA, about five-fold greater than that of classic PCR assays [26].

Just like PCR, most of LAMP techniques are just suitable for single gene detection. Recently, Randhawa et al. (2013) [7] reported the simultaneous screening of three GMO elements including P-35S, Figwort Mosaic Virus promoter (P-FMV) and the marker gene neomycin phosphotransferase II (nptII) in eight GMO event cottons [7] 
Table 1. The application of LAMP in GMO detection.

\begin{tabular}{|c|c|c|c|c|}
\hline \multirow{2}{*}{ Purpose } & \multirow{2}{*}{ Detected targets/events } & \multicolumn{2}{|l|}{ Detection limit } & \multirow{2}{*}{ Reference } \\
\hline & & Materials & LOD & \\
\hline \multirow{6}{*}{$\begin{array}{l}\text { Screening } \\
\text { common } \\
\text { elements }\end{array}$} & & $5 \%, 2 \%, 1 \%, 0.5 \%, 0 \%$ roundup ready soybeans & $0.5 \%$ & [22] \\
\hline & P-CaMV35S & Soya Roundup Ready ${ }^{\mathrm{TM}}$ GMO Reference Material & 10 copies & [23] \\
\hline & & MON15985 & 4 copies & [7] \\
\hline & T-nos & Soya Roundup Ready ${ }^{\mathrm{TM}}$ GMO Reference Material & 10 copies & [23] \\
\hline & P-nos & The plasmid pGreenII & 6 copies & [23] \\
\hline & P-FMV & MON88913 & 4 copies & [7] \\
\hline \multirow{6}{*}{$\begin{array}{c}\text { Screening } \\
\text { marker } \\
\text { genes }\end{array}$} & nptII & MON15985 & 4 copies & [7] \\
\hline & cry1Ab & Transgenic rice line KMD1 & $0.5 \%$ GM contents & [25] \\
\hline & cry2Ab & GM maize MON89034 & 5 copies & [26] \\
\hline & cry3A & GM maize MIR604 & 5 copies & [26] \\
\hline & pat & Plasmid pGreen0229 DNA & $0.65 \mathrm{fg} /$ tube & [24] \\
\hline & Phytase gene & Transgenic maize with phytase (BVLA 430101) & 30 copies & [31] \\
\hline \multirow{19}{*}{$\begin{array}{l}\text { Event } \\
\text { specific } \\
\text { detection }\end{array}$} & \multirow{6}{*}{ Rice } & \multirow{2}{*}{ TT51-1 } & $0.01 \%$ & [29] \\
\hline & & & 15 copies & [32] \\
\hline & & KMD1 & $0.01 \%$ & [29] \\
\hline & & \multirow{2}{*}{ KF6 } & $0.005 \%$ & [29] \\
\hline & & & 10 copies & [32] \\
\hline & & T1C-19 & 10 copies & [32] \\
\hline & \multirow{7}{*}{ Maize } & DAS-59122-7 & 4 copies & [27] \\
\hline & & $\mathrm{T} 25$ & 4 copies & [27] \\
\hline & & BT176 & 4 copies & [27] \\
\hline & & TC1507 & 4 copies & [27] \\
\hline & & BT11 & 4 copies & [27] \\
\hline & & MON863 & 4 copies & [27] \\
\hline & & MON810 & 40 copies & [27] \\
\hline & \multirow{2}{*}{ Soybean } & GTS 40-3-2 & 4 copies & [13] \\
\hline & & MON89788 & 4 copies & [13] \\
\hline & Cotton & cry1 $\mathrm{A}(\mathrm{b})$ gene and chi gene in $\mathrm{T}_{2}$ transgenic cotton & $10^{-8}$ dilutions of chi gene & [28] \\
\hline & Wheat & B73-6-1 & 6 copies & {$[30]$} \\
\hline & \multirow{2}{*}{ Oilseed rape (OSR) } & MS8 & 8 copies & [23] \\
\hline & & RF3 & 8 copies & [23] \\
\hline
\end{tabular}

These result implied a high potential for the application of LAMP in GMO detection, in a single or multiplex manner.

\subsection{Detection of GM Events}

LAMP is increasingly used for the detection of GM events. It was successfully used for the detection of two GM soybean events (GTS 40-3-2 and MON89788) in 2010 [13]. The LAMP limit of detection (LOD) of GTS 40-3-2 was 4 copies; more sensitive than that of conventional PCR, in which the LOD of GTS 40-3-2 was 40 copies and the LAMP LOD of MON89788 was 8 copies.

The identification of seven GM maize events (DAS-59122-7, T25, Bt176, TC1507, MON810, Bt11, and MON863) with LAMP was reported in 2011, and the LOD was as low as four copies of maize haploid genomic DNA, with the exception of MON 810 [27]. In addition, LAMP has been used to detect GM cottons [28], the sensitivity of LAMP was found to be 100 -fold higher than that of PCR.

In 2012, a visual and rapid LAMP method has been developed for three GM rice events (KMD1, TT51-1, and KF6) [29], three most famous transgenic Bt rice lines developed in China.

In 2013, wheat B73-6-1 has also been detected by LAMP [30]. LOD of the visual LAMP assays was about 6 
copies of haploid wheat-genomic DNA.

Recently in 2014, phytase gene in GM maize was detected within less than 20 min using LAMP, and its sensitivity was about 30 copies of phytase genomic DNA, which is 30 times more than that of conventional PCR method. Its limit of quantification (LOQ) was 60 copies [31].

\section{Disadvantages or Problems of LAMP}

No GMO detection method is perfect, neither is LAMP. LAMP is undoubtedly much sensitive than other GMO detection methods; however, obvious shortages of it also emerge along the analysis process. Firstly, LAMP needs at least 4 primers target 6 distinct regions of target gene. Therefore, the LAMP primer design is timeconsuming and difficult, particularly when the available target sequence is too short. Usually, the specific LAMP primer design website can generate hundreds of primers, and it take a long time to screen for suitable primer pairs. Secondly, the products are easily contaminated by aerosol when the lid is taken off because of the high sensitivity of LAMP. Thirdly, LAMP positive reaction displays ladder-like bands on the gel, and it is difficult to distinguish the non-specificity amplification from specificity amplification and false positive results are easy to appear; and fourthly, the LAMP products can't be sequenced for the confirmation of the amplification of target sequences. In addition, when the quantification of the amplification products are needed, heavy instrument such as fluorescent quantitation PCR equipment or turbidity meter has to be invested. Furthermore, in the case of the result visualization by inflorescent dying, current SYBR is generally an inhibitor of the template DNA, therefore, it is not possible to add the dying before the amplification. But, when addition of dying after the amplification, the lid of reaction tube has to be open, and which generates cross-contamination. All those disadvantages impeded the application of LAMP in GMO detection, specifically for on-spot detection.

\section{Future Improvements for LAMP to Be Practical for On-Site GMO Detection}

LAMP detection procedure consists mainly of three parts, namely nucleic acid extraction, LAMP amplification and result judgments. Any improvements in each of these three parts will contribute to the applicability and of LAMP to on-site GMO detection.

\subsection{DNA Extraction}

The development of a rapid and efficient DNA extraction method is essential for LAMP based on-site GMO detection. DNA extraction and purification methods widely used in the laboratory, such as, CTAB method, SDS method, and commercial kits including DNeasy Plant Mini Kit from Qiagen, do not fit for this purpose. Zhang et al. [32] have tested the applicability of visual LAMP assay to on-site detection of GM rice, using a creative DNA extraction device included a silica gel membrane filtration column and a modified syringe. It is simple, fast, and effective for on-site detection of GM rice T1c-19, TT51-1 and Kefeng 6 [32], showing for the first time that LAMP is potential in reaching the demand for novel technologies for GMO on-site detection. However, its defectiveness regarding to high throughput needs to be overcome with novel improvements on the device. Specific centrifuge-free and high throughout DNA extraction kits for various tissues such as leaves, developing seeds or flowers needs to be developed in the future.

\subsection{LAMP Amplification}

A highly efficient, low cost and less time-consuming LAMP amplification facility is one of the key points for its application on GMO on-site testing. For LAMP amplification of target DNA on-field, an isothermal apparatus that can reach to and maintain $60^{\circ} \mathrm{C}-65^{\circ} \mathrm{C}$ is indispensable. In the laboratory, LAMP amplification of target DNA can be achieved through isothermal equipment such as a water bath pot or a thermal cycler. However, when testing on-site without electricity, novel portable isothermal apparatus independent on direct electricity resource will be essential. In order to apply LAMP to on-site GMO detection, chargeable isothermal equipment dependent on battery or vehicle-mounted heater would be also useful alternatives.

\subsection{LAMP Result Judgments}

For an effective on-site GMO detection, the identification and quantification of LAMP products should also be 
rapid and sensitive. In the laboratory, amplified products can be observed directly by naked eye in the reaction tube by adding SYBR Green I stain or picogreen dye, or by gel electrophoresis under UV trans-illuminator to see a ladder-like structure, or by turbidity assay based on chemical reactions [15]-[17]. Currently, it seems that only fluorescent dye is feasible for on-site application. In this case, due to the inhibitory effect of SYBR Green I dye on isothermal amplification, the dye has to be added after the reaction, the opening of the lid of reaction tube to add the dye readily results in contamination. Zhang et al. [32] reported a novel method to avoid opening the lid of reaction tube by encapsulating SYBR Green I fluorescent dye into heat-melted microcrystalline wax capsules. Therefore, after the isothermal amplification, by raising the temperature up to the melt point of the microcrystalline wax, the SYBR Green I dye is released from the melted wax and reacts with amplification products to give fluorescent signal. Although it is creative, the need for the management of two temperature phases reduces significantly its practical utility for on-site GMO detection. Therefore, future identification of LAMP amplification products should focus on the development of new fluorescent dyes that do not inhibit the LAMP enzyme; in this case, the dye can be added together with the reaction mixture before amplification, which eliminates the management of reaction temperature as well. Additional focuses include the development of sensitive and portable turbidity sensors based on chemical reactions during LAMP amplification and the development of LAMP enzymes compatible with SYBR Green I dye.

\section{Future Perspectives}

With the increasing number and complexity of GM events globally, judging for the presence or absence of GM contents in crops with high specificity and sensitivity, cost-effective methods, has becoming a pressing need. LAMP proves to be more sensitive than conventional PCR methods, and its result is visible; therefore, it is of highly potential to be an alternative to conventional PCR for GMO detection, especially as a field detection alternative in scenes where a fast screen is needed while it lacks complex laboratory equipment. The practicability of LAMP will facilitate GMO detection in the laboratory and also on spot, if it is combined with other techniques, such as a simple and rapid DNA extraction device, a portable isothermal apparatus or a simple heating block. And from a long-term perspective, strategies and methods to avoid the opening of the cap of the reaction tubes will make the LAMP method more convenient for GMO detection in both lab and outdoors. Further emphasis should also go to the development of high throughput LAMP methods such as multiple LAMP methods. The last but not least is its capacity for automatic quantification. With the current characteristics, together with the future improvement in the assay, we strongly believe that LAMP will contribute significantly to GMO detection both on laboratory and on-site.

\section{Acknowledgements}

This work was partially supported by the National Transgenic Plant Special Fund (2013-2014ZX08012-002) and EU FP7 project DECATHLON (613908).

\section{Conflict of Interest}

The authors declare that there are no conflicts of interest.

\section{References}

[1] James, C. (2011) Global Status of Commercialized Biotech/GM Crops: 2011. Executive Summary. ISAAA Brief 43.

[2] Smythe, S., Kerr, W.A. and Davey, K.A. (2006) Closing Markets to Biotechnology: Does It Pose an Economic Risk If Markets Are Globalised? International Journal of Technology and Globalisation, 2, 377-389. http://dx.doi.org/10.1504/IJTG.2006.011923

[3] Broeders, S.R.M., Keersmaecker, S.C.J. and Roosens, N.H.C. (2012) How to Deal with the Upcoming Challenges in GMO Detection in Food and Feed. BioMed Research International, 2012, Article ID: 402418.

[4] Bonfini, L. (2002) Review of GMO Detection and Quantification Techniques. Institute for Health and Consumer Protection. Food Products and Consumer Goods Unit, 1-74.

[5] Zhang, D.B. and Guo, J.C. (2011) The Development and Standardization of Testing Methods for Genetically Modified Organisms and Their Derived Products. Journal of Integrative Plant Biology, 53, 539-551. http://dx.doi.org/10.1111/j.1744-7909.2011.01060.x 
[6] Suchitra, K. and Sher, A. (2013) Genetically Modified Crops: Detection Strategies and Biosafety Issues. Gene, 522, 123-132. http://dx.doi.org/10.1016/j.gene.2013.03.107

[7] Randhawa, G.J., Singh, M. and Morisset, D. (2013) Loop-Mediated Isothermal Amplification: Rapid Visual and RealTime Methods for Detection of Genetically Modified Crops. Journal of Agricultural and Food Chemistry, 61, 1133811346. http://dx.doi.org/10.1021/jf4030085

[8] James, C. (2013) Global Status of Commercialized Biotech/GM Crops: 2013; ISAAA Brief No. 46. International Service for the Acquisition Agri-Biotech Applications (ISAAA), Ithaca.

[9] Gruere, G.P., Carter, C.A. and Hossein, F. (2009) Explaining International Differences in Genetically Modified Food Labeling Policies. Review of International Economics, 17, 393-408. http://dx.doi.org/10.1111/j.1467-9396.2008.00788.x

[10] Arne, H.J. (2009) Testing for Genetically Modified Organisms (GMOs): Past, Present and Future Perspectives. Biotechnology Advances, 27, 1071-1082. http://dx.doi.org/10.1016/j.biotechadv.2009.05.025

[11] Marmiroli, N., Maestri, E., Gullì, M., Malcevschi, A., Peano, C., Bordoni, R. and De Bellis, G. (2008) Methods for Detection of GMOs in Food and Feed. Analytical and Bioanalytical Chemistry, 392, 369-384. http://dx.doi.org/10.1007/s00216-008-2303-6

[12] Arne, H.J., Marc, D.L. and Guy, V.D.E. (2006) Coherence between Legal Requirements and Approaches for Detection of Genetically Modified Organisms (GMOs) and Their Derived Products. Journal of Agricultural and Food Chemistry, 54, 2799-2809. http://dx.doi.org/10.1021/jf052849a

[13] Guan, X.Y., Guo, J.C., Shen, P., Yang, L.T. and Zhang, D.B. (2010) Visual and Rapid Detection of Two Genetically Modified Soybean Events Using Loop-Mediated Isothermal Amplification Method. Food Analytical Methods, 3, 313320. http://dx.doi.org/10.1007/s12161-010-9132-X

[14] Notomi, T., Okayama, H., Masubuchi, H., Yonekawa, T., Watanabe, K., Amino, N. and Hase, T. (2000) Loop-Mediated Isothermal Amplification of DNA. Nucleic Acids Research, 28, e63. http://dx.doi.org/10.1093/nar/28.12.e63

[15] Mori, Y. and Notomi, T. (2009) Loop-Mediated Isothermal Amplification (LAMP): A Rapid, Accurate, and CostEffective Diagnostic Method for Infectious Diseases. Journal of Infection and Chemotherapy, 15, 62-69. http://dx.doi.org/10.1007/s10156-009-0669-9

[16] Fu, S.J., Qu, G.G., Guo, S.J., Ma, L., Zhang, N., Zhang, S.L., Gao, S.Y. and Shen, Z.Q. (2011) Applications of LoopMediated Isothermal DNA Amplification. Applied Biochemistry and Biotechnology, 163, 845-850. http://dx.doi.org/10.1007/s12010-010-9088-8

[17] Mori, Y., Kanda, H. and Notomi, T. (2013) Loop-Mediated Isothermal Amplification (LAMP): Recent Progress in Research and Development. Journal of Infection and Chemotherapy, 19, 404-411. http://dx.doi.org/10.1007/s10156-013-0590-0

[18] Francois, P., Tangomo, M., Hibbs, J., Bonetti, E.J., Boehme, C.C., Notomi, T., Perkins, M.D. and Schrenzel, J. (2011) Robustness of a Loop Mediated Isothermal Amplification Reaction for Diagnostic Applications. FEMS Immunology and Medical Microbiology, 62, 41-48. http://dx.doi.org/10.1111/j.1574-695X.2011.00785.X

[19] Kiddle, G., Hardinge, P., Buttigieg, N., Gandelman, O., Pereira, C., McElgunn, C., Rizzoli, M., Jackson, R., Appleton, N., Moore, C., Tisi, L. and Murray, J. (2012) GMO Detection Using a Bioluminescent Real Time Reporter (BART) of Loop Mediated Isothermal Amplification (LAMP) Suitable for Field Use. BMC Biotechnology, 12, 15. http://dx.doi.org/10.1186/1472-6750-12-15

[20] Tomita, N., Mori, Y., Kanda, H. and Notomi, T. (2008) Loop-Mediated Isothermal Amplification (LAMP) of Gene Sequences and Simple Visual Detection of Products. Nature Protocols, 3, 877-882. http://dx.doi.org/10.1038/nprot.2008.57

[21] Asiello, P.J. and Baeumner, A.J. (2011) Miniaturized Isothermal Nucleic Acid Amplification: A Review. Lab on a Chip, 11, 1420-1430. http://dx.doi.org/10.1039/c0lc00666a

[22] Fukuta, S., Mizukami, Y., Ishida, A., Ueda, J., Hasegawa, M., Hayashi, I., Hashimoto, M. and Kanbe, M. (2004) RealTime Loop-Mediated Isothermal Amplification for the CaMV-35S Promoter as a Screening Method for Genetically Modified Organisms. European Food Research and Technology, 218, 496-500. http://dx.doi.org/10.1007/s00217-003-0862-5

[23] Lee, D., La, M.M., Allnutt, T. and Powell, W. (2009) Detection of Genetically Modified Organisms (GMOs) Using Isothermal Amplification of Target DNA Sequences. BMC Biotechnology, 9, 7. http://dx.doi.org/10.1186/1472-6750-9-7

[24] Chen, J., Huang, C., Zhang, X., Yu, R. and Wu, Z. (2011) Detection of Herbicide-Resistant Maize by Using LoopMediated Isothermal Amplification of the Pat Selectable Marker Gene. African Journal of Biotechnology, 10, 1705517061 . 
[25] Li, Q., Fang, J., Liu, X., Xi, X., Li, M., Gong, Y. and Zhang, M. (2013) Loop-Mediated Isothermal Amplification (LAMP) Method for Rapid Detection of cry $1 A b$ Gene in Transgenic Rice (Oryza sativa L.). European Food Research and Technology, 236, 589-598. http://dx.doi.org/10.1007/s00217-013-1911-3

[26] Li, F.W., Yan, W., Long, L.K., Qi, X., Li, C.C. and Zhang, S.H. (2014) Development and Application of LoopMediated Isothermal Amplification Assays for Rapid Visual Detection of cry2Ab and cry3A Genes in GeneticallyModified Crops. International Journal of Molecular Sciences, 15, 15109-15121. http://dx.doi.org/10.3390/ijms150915109

[27] Chen, L.L., Guo, J.C., Wang, Q.D., Kai, G.Y. and Yang, L.T. (2011) Development of the Visual LAMP Assays for Seven Genetically Modified Maize Events and Their Application in Practical Samples Analysis. Journal of Agricultural and Food Chemistry, 59, 5914-5918. http://dx.doi.org/10.1021/jf200459s

[28] Niloofar, R., Ali, H., Masoud, T. and Aboubakr, M. (2011) Rapid Identification of Transgenic Cotton (Gossypium hirsutum L.) Plants by Loop-Mediated Isothermal Amplification. Czech Journal of Genetics and Plant Breeding, 47, 140148.

[29] Chen, X.Y., Wang, X.F., Jin, N., Zhou, Y., Huang, S.N., Miao, Q.M., Zhu, Q. and Xu, J.F. (2012) Endpoint Visual Detection of Three Genetically Modified Rice Events by Loop-Mediated Isothermal Amplification. International Journal of Molecular Sciences, 13, 14421-14433. http://dx.doi.org/10.3390/ijms131114421

[30] Cheng, Y., Zhang, M.H., Hu, K., Sun, F.D., Tao, R., Gao, X.J. and Luan, F.X. (2013) Loop-Mediated Isothermal Amplification for the Event-Specific Detection of Wheat B73-6-1. Food Analytical Methods, 7, 500-505. http://dx.doi.org/10.1007/s12161-013-9718-1

[31] Huang, X., Chen, L.L., Xu, J.M., Ji, H.F., Zhu, S.F. and Chen, H.J. (2014) Rapid Visual Detection of Phytase Gene in Genetically Modified Maize Using Loop-Mediated Isothermal Amplification Method. Food Chemistry, 156, 184-189. http://dx.doi.org/10.1016/j.foodchem.2014.01.102

[32] Zhang, M., Liu, Y.N., Chen, L.L., Quan, S., Jiang, S.M., Zhang, D.B. and Yang, L.T. (2013) One Simple DNA Extraction Device and Its Combination with Modified Visual Loop-Mediated Isothermal Amplification for Rapid On-Field Detection of Genetically Modified Organisms. Analytical Chemistry, 85, 75-82. http://dx.doi.org/10.1021/ac301640p 
Scientific Research Publishing (SCIRP) is one of the largest Open Access journal publishers. It is currently publishing more than 200 open access, online, peer-reviewed journals covering a wide range of academic disciplines. SCIRP serves the worldwide academic communities and contributes to the progress and application of science with its publication.

Other selected journals from SCIRP are listed as below. Submit your manuscript to us via either submit@scirp.org or Online Submission Portal.
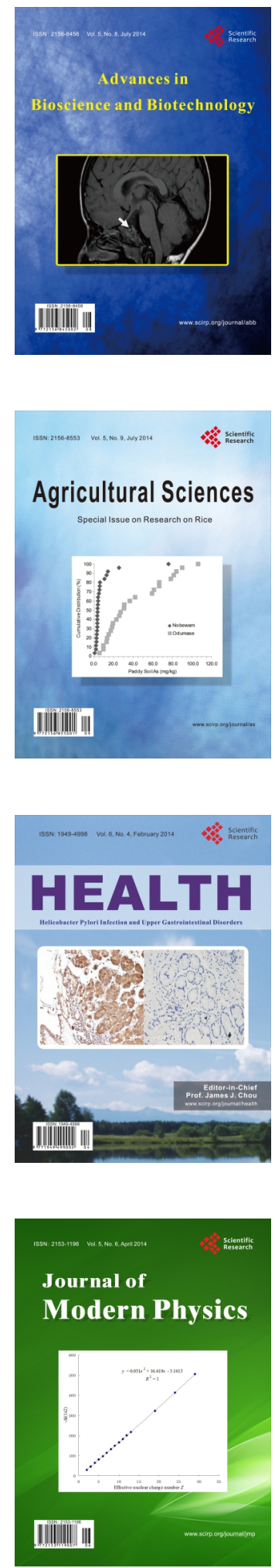
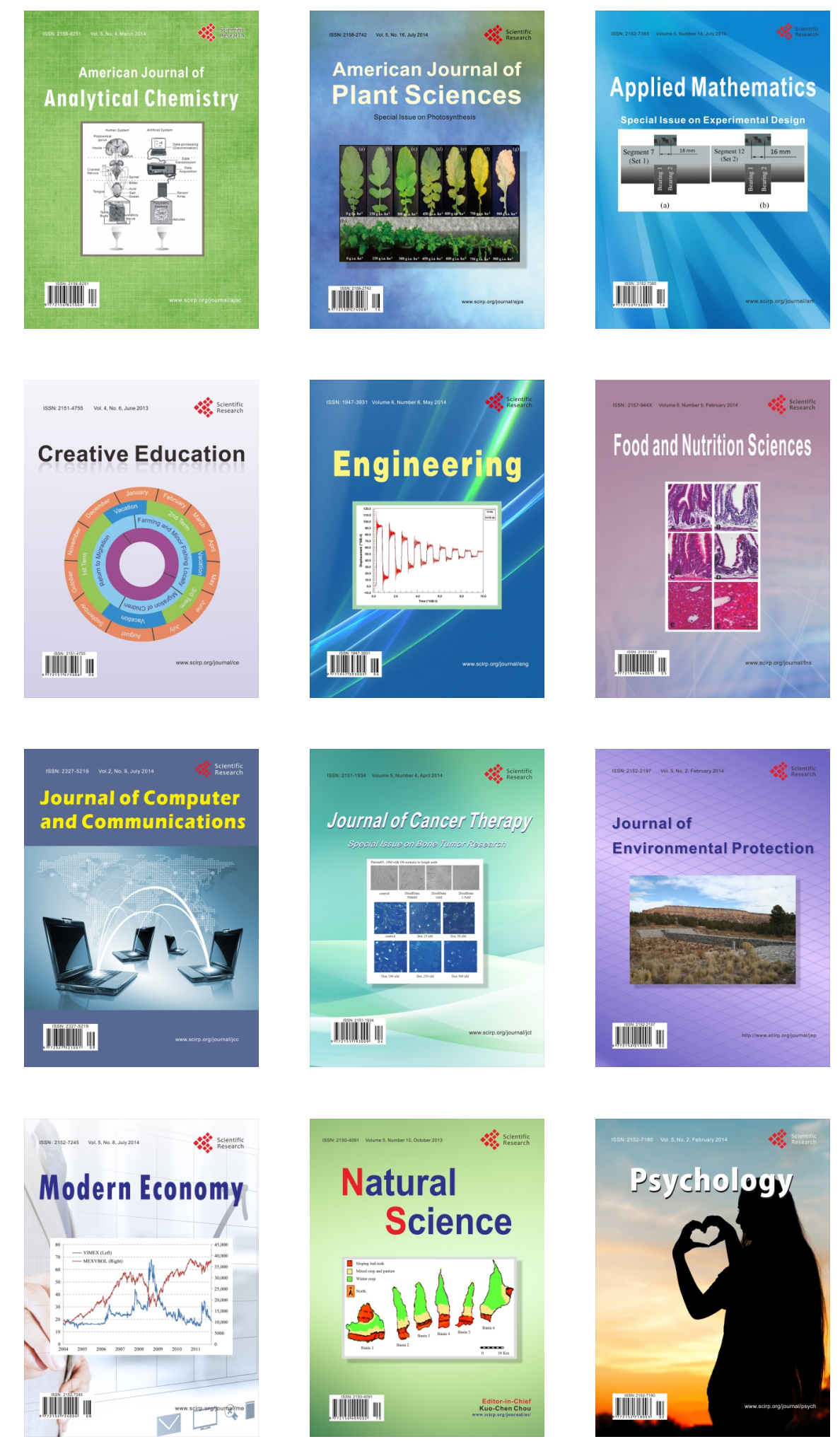